\title{
Synthesis of sources with Markovian features
}

\section{G. Martínez-Niconoff gmartin@inaoep.mx}

\section{P. Martínez-Vara}

\section{E. Andrés-Zarate}

\section{J. Silva-Barranco}

\section{J. Munoz-Lopez}

Instituto Nacional de Astrofísica Óptica y Electrónica, INAOE, Dpto. de Óptica, Puebla, México

Benemérita Universidad Autónoma de Puebla, BUAP, Dpto. de Ingeniería. Puebla, México

Instituto Nacional de Astrofísica Óptica y Electrónica, INAOE, Dpto. de Óptica, Puebla, México

Instituto Nacional de Astrofísica Óptica y Electrónica, INAOE, Dpto. de Óptica, Puebla, México

We realize the design and synthesis of partially coherent secondary sources illuminating an ensemble of mobile transmittances in a tandem array. The motion states of the transmittances are random variables which are synchronized by means of the conditional probability density function whose control allows generating sources whose light emission corresponds with a Markov chain. The experimental results are shown. [DOI: http://dx.doi.org/10.2971/jeos.2013.13005]

Keywords: Coherence, stochastic process

\section{INTRODUCTION}

In recent times, in almost all the optical areas, it has become necessary to synthesize partially coherent sources. For example, in microscopy it is necessary to have low coherence sources in order to control the diffraction effects, in communication system, coherence modulators have been proposed to drive and send a great volume of information. In metamaterials, it is necessary to use a partially coherent field to induce tuneable resonance effects to generate self-transparency, in plasmon optics is necessary to transfer the partially coherence features to surface plasmon fields in order to generate long range surface plasmon modes [1]-[5].

A great part of coherence theory is supported by the fact that primary sources can be visualized as formed by a set of point sources, where all of them are statistically independent. This means that light emerging from each point does not influence the emission of the neighbouring points. For incandescent and discharge sources the emission time from each point is typically of $10^{-8} \mathrm{~s}$. Then, for smaller times, we can expect certain correlation between the light emerging from each point and the hypothesis on statistical independence is no longer valid. These effects become evident in pulsed lasers, where the pulse width may be in the range of nano/femto-seconds and the coherence length is extremely short. In order to analyse this effect, correlation processes of third and fourth order are necessary. In this way, the Markov process can be a suitable model to describe the coherence structure of light, this is because in a Markov process the future states depends of the past and presents states. The definition of a Markov process is given by the conditional probability density function
$P\left(x_{i+p}, t_{i+p}, \ldots ; x_{i+1}, t_{i+1} \mid x_{i}, t_{i}, \ldots ; x_{1}, t_{1}\right)$, where $x_{i}$ are the states of a random variable at given time $t_{i}$ [6], for the present study $x_{i}$ represents the motion states of the boundary condition for the optical field and it is applied to the synthesis of a new kind of sources whose light emission depends on its history, corresponding to the Markov process.

The principal feature of these sources is that they have a substructure that can be controlled. The study is performed in the context of the physical optics, where, in general, the control of light is obtained by the design and illumination of objects that have a transmittance functions $T(x, y)$, corresponding to the boundary condition of the optical field. In order to induce partially coherent features, it is necessary to employ time-dependent transmittances [7]-[10]. We propose to use separable transmittances of the form $T(x, y, t)=T_{1}\left(x, y, t_{1}\right) T_{2}\left(x, y, t_{2}\right) \ldots T_{N}\left(x, y, t_{N}\right)[11]$.

The reason to use this transmittance is that we can to control the number $N$ of transmittances $T_{i}$ and put each one in independent motion state, also the movements can be time ordering in a probabilistic sense. With this configuration we generate optical fields whose amplitude correlation function is easily controlled. The motion states of each transmittance are considered as random variables, having two possible values " 1 " with probability $p$ or " 0 " with probability $q$, meaning that each transmittance $T_{i}$ is static or in motion respectively, however the kind of movements implemented are completely deterministic and for experimental convenience they are considered as harmonic oscillating movements. With this 
configuration it is possible to generate correlation functions of several orders, where the motion state of one of the transmittances called, transmittance of control, determinates the motion states of the other transmittances. The diffraction field on the Fraunhofer plane is interpreted as a secondary source where the transversal structure depends on the motions state of each constitutive transmittance, in consequence the light emission from each point on source is time ordered in a probabilistic sense, corresponding this to a Markov process.

When the motions of the constitutive transmittances are statistically independent, the optical field is a random process whose coherence parameters depend on the product of the individual probability density functions. The representation of mobile transmittance, in frequency space implies convolution functions among time-dependent functions for the phase term. The diffraction fields are subjected to a time-dependent amplitude/energy redistribution that carries on the manifestation of the partially coherent features and the traditional study of coherence can be applied.

The source with Markovian features is obtained when the motions of one of the transmittances is correlated with the motion of the second one and so on. This behavior is characterized by means of the joint probability density function. In this context, the simplest case is obtained when the Markov process corresponds with a Markov chain whose analysis is the goal pursuit of the present study. We show that the structure of the secondary source depends on the initial state of the first transmittance, whose transition to the motion states of the following transmittances generates a set of allowed states whose final result is associated to the mean intensity of the source.

In this paper we synthesize partially coherent secondary sources by controlling the light propagation through a set of mobile transmittances in a tandem array, where the motion state of the constitutive transmittance are time ordered by means of a conditional probability density function.

\section{SYNTHESIS OF COHERENT SECONDARY SOURCES}

With the purpose to have a visual reference to the optical field associated to static transmittances, we describe the diffraction field on the Fraunhofer plane for a set of transmittances illuminated with a plane wave, the optical field is given by the Fourier transform of the transmittance function and $z=0$ define the plane of the secondary source whose amplitude representation is given by

$$
\phi(u, v, z=0)=\int_{-\infty}^{\infty} \int_{-\infty}^{\infty} \prod T_{i}(x, y) e^{-i 2 \pi(x u+y v)} d x d y,
$$

where $u=x_{0} / \lambda f, v=y_{0} / \lambda f$. In Eq. (1) we have omitted a non-relevant term. This optical field is generated on the focal plane of a lens as was described by Goodman [12] and the optical system is sketched in Figure 1. The mathematical representation for the diffraction field corresponds with the convolution function between the Fourier transform associated to each transmittance represented

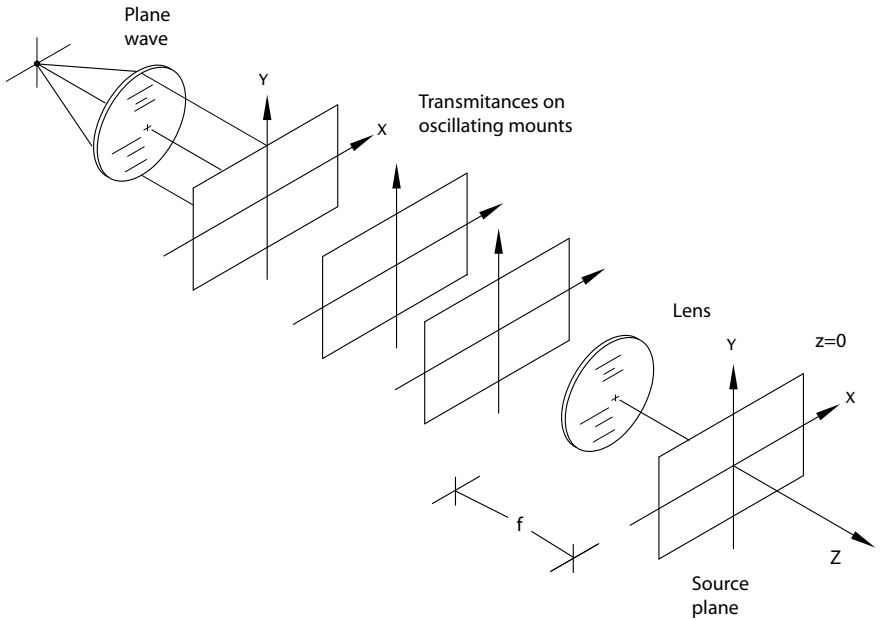

FIG. 1 The experimental set-up. The lens images the virtual source plane placed at infinity and the source becomes real. The transmittances are placed on an oscillating mount controlled with a signal generator.
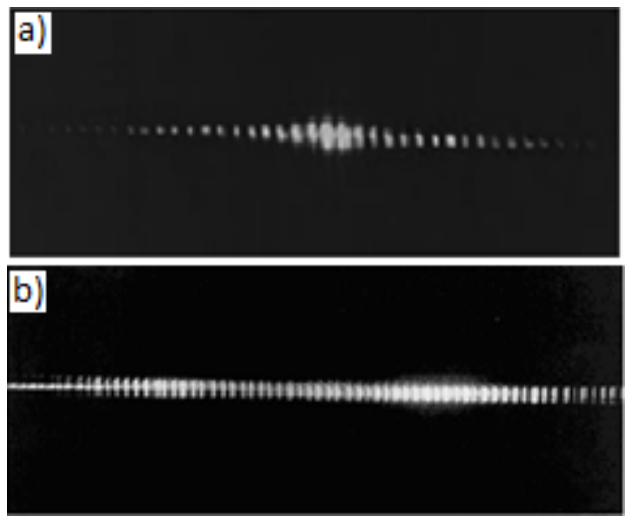

FIG. 2 Secondary coherent source obtained in $z=0$ for: (a) a single Ronchi grating. In (b) for an ensemble of six linear Ronchi gratings. The convolution function has associated a transversal energy redistribution, the optical field is completely coherent, and all the transmittances are static. The period of each grating is 100 lines per inch and each transmittance was recorded on high resolution plate HRP.

by $\phi(u, v, z=0)=T_{1}(u, v) \otimes T_{2}(u, v) \otimes \ldots \otimes T_{n}(u, v) . \quad$ In Figure 2, we show the experimental results for an ensemble of linear gratings, in this case, the convolution function explain the energy redistribution.

\section{SYNTHESIS OF PARTIALLY COHERENT SECONDARY SOURCES}

Partially coherent fields imply random temporal fluctuations; these may be induced and controlled by considering a temporal dependence on the transmittance function. A suitable system to be implemented in the laboratory consists of a set of mobile transmittances in a tandem array. The analysis presented here considers motion only along one coordinate. The extension to two coordinates is straightforward. Their representation takes the form

$$
\bar{T}(x, t)=\prod_{i=1}^{n} \bar{T}_{i}\left(x-f_{i}(t)\right) .
$$

Using the displacement property of the Fourier transform and considering it for an arbitrary time $t$, the intensity of the sec- 
ondary source is

$$
\begin{aligned}
I(u, z=0, t)= & \mid T_{1}(u) e^{i 2 \pi u f_{1}(t)} \otimes T_{2}(u) e^{i 2 \pi u f_{2}(t)} \\
& \left.\otimes \ldots \otimes T_{n}(u) e^{i 2 \pi u f_{n}(t)}\right|^{2} .
\end{aligned}
$$

We have that the convolution generates energy redistribution and the motions induce a time-dependent phase term on each point of the source which makes the difference with the coherent case, consequently, the source presents partial coherence features that will be analyzed in brief.

As an interesting example, let us consider two circular mobile transmittances in a one-dimensional harmonic movement along the $\mathrm{x}$ coordinate. The temporal functions proposed are given by $f_{1}(t)=\alpha \cos w_{1} t, f_{2}(t)=\alpha \cos w_{2} t, \alpha, \beta$ are the amplitudes and $w_{1,2}$, are the frequencies of each oscillation. The mean intensity of the source is

$$
\begin{aligned}
& \langle I(u, z=0)\rangle \\
& \quad=\left\langle\left|T_{1}(u) \exp \left(i 2 \pi u f_{1}(t)\right) \otimes T_{2}(u) \exp \left(i 2 \pi u f_{2}(t)\right)\right|^{2}\right\rangle \\
& \quad=\lim _{T \rightarrow \infty} \frac{1}{T} \int_{-T / 2}^{T / 2}\left|T_{1}(u) e^{i 2 \pi u f_{1}(t)} \otimes T_{2}(u) e^{i 2 \pi u f_{2}(t)}\right|^{2} d t .
\end{aligned}
$$

Writing the expression for the convolution function explicitly and reversing the order of integration, we find that the mean intensity acquires the form

$$
\begin{aligned}
& \langle I(u, z=0)\rangle=\left\langle|T(u, t,)|^{2}\right\rangle \\
& =\int_{-\infty}^{\infty} \int_{-\infty}^{\infty}\left\langle T_{1}(h) T_{2}(h-u) T_{1}^{*}(s) T_{2}^{*}(s-u)\right\rangle M(h, s, u) d h d s,
\end{aligned}
$$

which corresponds to a double convolution function, and

$$
\begin{aligned}
& M(h, s, u) \\
& =\lim _{T \rightarrow \infty} \frac{1}{T} \int_{0}^{T} \exp \left(i 2 \pi u(h-s)\left(\alpha \cos w_{1} t+\beta \cos w_{2} t\right)\right) d t .
\end{aligned}
$$

Using the Jacobi identity to transform the exponential [13] and considering the special case of equal frequencies [14], we obtain an approximate expression for Eq. (6)

$$
M(h, s, u) \approx J_{0}(2 \pi u(\alpha)(h-s)) J_{0}(2 \pi u(\beta)(h-s)) .
$$

The mean intensity distribution of the source acquires the form

$$
\begin{aligned}
\langle I(u, z= & 0)\rangle \\
= & \int_{-\infty}^{\infty} \int_{-\infty}^{\infty}\left\langle T_{1}(h) T_{2}(h-u) T_{1}^{*}(s) T_{2}^{*}(s-u)\right\rangle \\
& \times J_{0}(2 \pi u(\alpha)(h-s)) J_{0}(2 \pi u(\beta)(h-s) d h d s .
\end{aligned}
$$

From the last expression, we deduce that the motions of the transmittances generate a curve (represented by the product of the Bessel functions) which is convolved with the irradiance distribution generated when the transmittances are static. The last statement explains the experimental results shown in Figure 3(a) we show the diffraction field for two static circular transmittances of the same diameter. Superimposed on this figure we schematically show the product of the Bessel functions generated by the oscillations with different amplitude
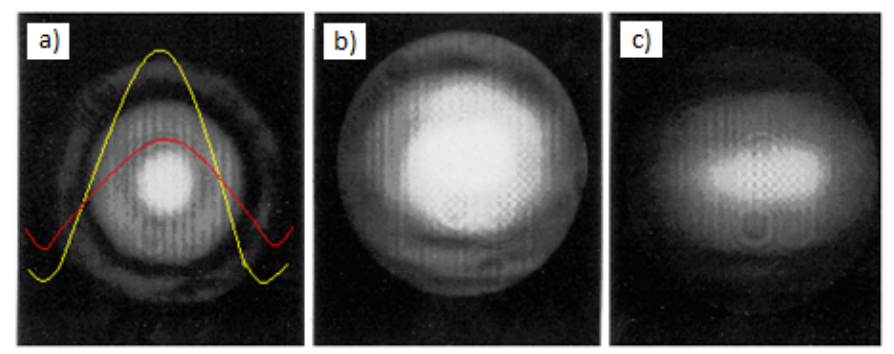

FIG. 3 Experimental results for the synthesis of partially coherent sources. In (a), we see a completely coherent source generated by the diffraction of two static circular transmittances in tandem. The radius of each circle is approximately $2 \mathrm{~mm}$. In (b) and (c) each transmittance is in harmonic movement with the same frequencies, approximately $300 \mathrm{~Hz}$, but different amplitude, $\alpha=\beta=2 \mathrm{~mm}, \alpha=2 \mathrm{~mm}, \beta=3 \mathrm{~mm}$. The yellow and red lines in (a) represent the product of the two zero order Bessel functions. Each transmittance was placed on an oscillating mount controlled by a signal generator.

values represented by $\alpha, \beta$, and in Figure 3(b) and (c) we show the effect of the convolution between the coherent source and each curve for two slightly different amplitudes. The coherence parameters of the source can be obtained following the Wolf formalism $[6,15]$, this implies integrals hard to calculate. We avoid the explicit calculus of this, using the fact that coherence features are manifested by the redistribution energy, that is, the radiometric features of the optical field are modified. Then, it is possible to compare the radiometric fluctuations of the partially coherent source to those completely coherent sources. This propose will be applied later to describe the coherence parameters of Markovian sources. To evaluate the coherence of the optical field, we use a correlation coefficient similar to the visibility function given by

$$
\gamma(x, y, z)=\frac{\left\langle I_{\mathcal{c}}(x, y, z)\right\rangle-\left\langle I_{i n c}(x, y, z)\right\rangle}{\left\langle I_{\mathcal{c}}(x, y, z)\right\rangle+\left\langle I_{i n c}(x, y, z)\right\rangle}
$$

This expression has the information of the energy redistribution generated in partially coherent sources. A similar expression to determinate the degree of polarization was proposed by Goodman [16].

In Eq. (9) $\left\langle I_{\mathcal{C}}(p)\right\rangle$ represents the mean irradiance on a point $p(x, y, z)$ when the transmittances are static (coherent case), $\left\langle I_{\text {inc }}(p)\right\rangle$ is the mean irradiance associated when the transmittances are moving (partially coherent case). From this representation the partially coherent features are detected from the redistribution energy. If the transmittances are non-mobile we have $\left\langle I_{\text {inc }}(p)\right\rangle=0$ and the correlation coefficient is $\gamma(p)=1$, corresponding with a completely coherent source. It must be noted that the correlation coefficient function can be negative. This occur because it is possible that on some points and $I_{\text {inc }} \neq 0$. This means that the optical fields are completely non-correlated, in this case, the correlation coefficient is $\gamma(p)=-1$. It must be noted that for static transmittances, that is, substituting the values $\alpha=\beta=0$, in Eq. (8), the correlation coefficient takes the value of 1 , which means that we have optical sources completely coherent. The correlation coefficient can be obtained from the experimental results shown in Figure 3, this is done by comparing the measurements of the irradiance on each point and substituting the values in Eq. (9). The diffraction field associated to mobile transmittances is dif- 


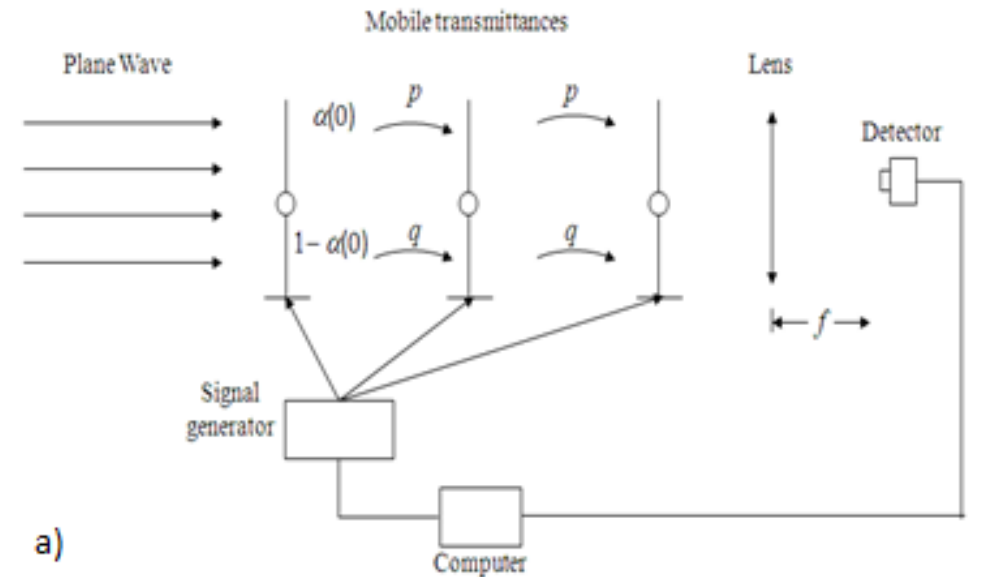

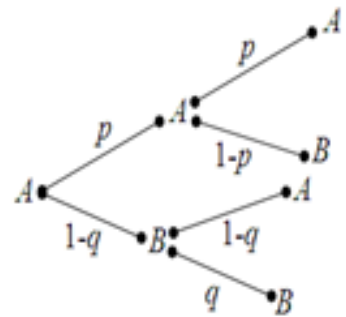

b)

FIG. 4 a) Schematic system to generate Markovian sources. b) Diagram kind tree to associate the transition probabilities for a Markov chain with two states $A$ and $B$.

ferent to static transmittances, generating redistribution energy, consequently modifies the radiometric features of the source according with modern radiometric theory of Wolf [6]. The propagation of the coherence features to other planes is described in the appendix.

\section{SECONDARY SOURCES WITH MARKOV CHAIN FEATURES}

As a generalization of the previous analysis, we describe the synthesis of light whose amplitude function $\phi\left(x, y, z=0, t_{i}\right)$ depends on its recent past state $\phi\left(x, y, z=0, t_{i-1}\right)$. The optical system is a set of $n$-transmittances in a tandem array. We can consider the system as a Markov chain with two states, letting the state " 1 " represents the mobile transmittance and the state " 0 " represents the static transmittance. $P\left(X_{i}=1\right)$ represents the probability that the $i$-th transmittance is moving. The stationary transition probabilities are given by

$$
P\left(X_{i+1}=1 \mid X_{i}=0\right)=p, P\left(X_{i+1}=0 \mid X_{i}=1\right)=q .
$$

The first expression in Eq. (10) represents the probability $p$ that $i+1$-th transmittance is moving if the $i$-th transmittance is static. The kind of motion of each transmittance is deterministic harmonic oscillations where all of them have the same amplitude and frequency. The mean irradiance distribution of the ensemble takes the form

$$
\begin{aligned}
\langle I(u, z & =0)\rangle \\
& =\frac{1}{N} \sum_{s=1}^{N(s)}\left|T_{1}(u) e^{i 2 \pi u f_{1}(t)} \otimes \ldots \otimes T_{n}(u) e^{i 2 \pi u f_{n}(t)}\right|^{2},
\end{aligned}
$$

where the super index $(s)$ represents the number of realizations and $0 \leq t \leq t_{f}$, such that $t_{f}$ is the time duration of each realization. The mean irradiance for each realization is obtained as follows, for a Markov chain, we know that the conditional probability density function takes the form [17], [18]

$$
\rho\left(X_{1}, \ldots, X_{n}\right)=\rho\left(X_{1}\right) \rho\left(X_{2} \mid X_{1}\right) \rho\left(X_{3} \mid X_{2}\right) \ldots \rho\left(X_{n} \mid X_{n-1}\right) .
$$

The mean intensity of each realization contains the information of the correlation process between the motions of the transmittances. From the Markov chain theory, the probability of the $n$-th transmittance being static is given by [17]

$$
P\left(X_{n}=0\right)=\frac{q}{p+q}+(1-p-q)^{n}\left(\alpha(0)-\frac{q}{p+q}\right),
$$

where $\rho\left(X_{1}\right)=\alpha(0)$ is the probability that the first transmittance is static. The motion state of the first transmittance determinates the motion state of the other transmittances, for this reason, the first transmittance is considered as the control transmittance. The probability that the $n$-th transmittance is mobile is given by

$$
P\left(X_{n}=1\right)=\frac{p}{p+q}+(1-p-q)^{n}\left(\alpha(1)-\frac{p}{p+q}\right)
$$

\section{EXPERIMENTAL SYNTHESIS OF THE MARKOV CHAIN SOURCES}

Here we describe the synthesis of the Markovian sources using three transmittances. The process has $2^{3}$ possible states, given by the set $\{i, j, k\}=\{(1,1,1),(1,1,0),(1,0,1),(1,0,0),(0,1,1)$, $(0,1,0),(0,0,1),(0,0,0)\}$. The first element of the set means that the three transmittances are moving, the second means that the first two transmittances are moving and the third remains static, and similarly for other elements. The experimental set up is sketched in Figure 4.

The time duration of each state is $t_{0}$ and the time duration of the realization is $T=N t_{0}$ where $N$ is the number of transmittances, and the diffraction field of the realization has associated the mean intensity $I_{i j k}$.

With the Markov property given by Eq. (12), and using Eqs. (13) and (14), the expression for the correlation for three transmittances is given by

$$
\begin{aligned}
& P\left(X_{1}=\alpha_{i}, X_{2}=\beta_{j}, X_{3}=\gamma_{q}\right) \\
& =P\left(X_{1}=\alpha_{i}\right) P\left(X_{2}=\beta_{j} \mid X_{i}=\alpha_{i}\right) P\left(X_{3}=\gamma_{q} \mid X_{2}=\beta_{j}\right),
\end{aligned}
$$

where $\alpha_{i}, \beta_{j}, \gamma_{q}$ can take two possible values, represented by $(0,1)$. The expression for the probability for case where all transmittances are statics is given by

$$
\begin{aligned}
P\left(X_{1}\right. & \left.=0, X_{2}=0, X_{3}=0\right) \\
& =P\left(X_{1}=0\right) P\left(X_{2}=0 \mid X_{1}=0\right) P\left(X_{3}=0 \mid X_{2}=0\right),
\end{aligned}
$$




\begin{tabular}{|c|c|c|c|c|}
\hline$P\left(X_{1}\right)$ & $P\left(X_{2}\right)$ & $P\left(X_{3}\right)$ & $P\left(X_{1}, X_{2}, X_{3}\right)$ & $\langle I\rangle$ \\
\hline$P\left(X_{1}=0\right)$ & $P\left(X_{2}=0\right)$ & $P\left(X_{3}=0\right)$ & $\alpha(0)(1-p)^{2}$ & $\left\langle I_{c}(0,0,0)\right\rangle$ \\
$P\left(X_{1}=0\right)$ & $P\left(X_{2}=1\right)$ & $P\left(X_{3}=0\right)$ & $\alpha(0) p q$ & $\left\langle I_{\text {inc }}(0,1,0)\right\rangle$ \\
$P\left(X_{1}=0\right)$ & $P\left(X_{2}=0\right)$ & $P\left(X_{3}=1\right)$ & $\alpha(0)(1-p) p$ & $\left\langle I_{\text {inc }}(0,0,1)\right\rangle$ \\
$P\left(X_{1}=1\right)$ & $P\left(X_{2}=0\right)$ & $P\left(X_{3}=0\right)$ & $(1-\alpha(0))(1-p) q$ & $\left\langle I_{\text {inc }}(1,0,0)\right\rangle$ \\
$P\left(X_{1}=1\right)$ & $P\left(X_{2}=0\right)$ & $P\left(X_{3}=1\right)$ & $(1-\alpha(0)) q p$ & $\left\langle I_{\text {inc }}(1,0,1)\right\rangle$ \\
$P\left(X_{1}=0\right)$ & $P\left(X_{2}=1\right)$ & $P\left(X_{3}=1\right)$ & $\alpha(0)(1-q) p$ & $\left\langle I_{\text {inc }}(0,1,1)\right\rangle$ \\
$P\left(X_{1}=1\right)$ & $P\left(X_{2}=1\right)$ & $P\left(X_{3}=0\right)$ & $(1-\alpha(0))(1-q) q$ & $\left\langle I_{\text {inc }}(1,1,0)\right\rangle$ \\
$P\left(X_{1}=1\right)$ & $P\left(X_{2}=1\right)$ & $P\left(X_{3}=1\right)$ & $(1-\alpha(0))(1-q)^{2}$ & $\left\langle I_{\text {inc }}(1,1,1)\right\rangle$ \\
\hline
\end{tabular}

TABLE 1 Triple correlation values for three transmittances.
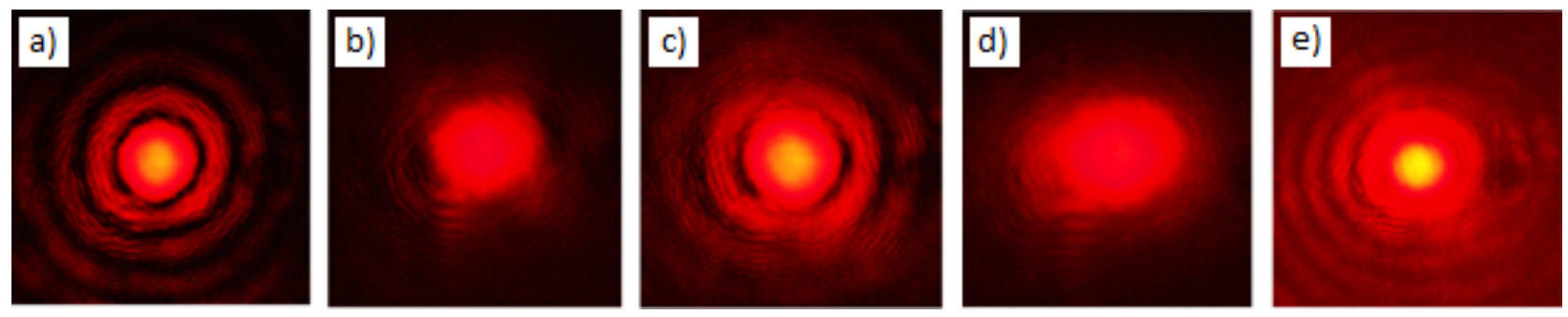

FIG. $5(\mathrm{a}),(\mathrm{b}),(\mathrm{c})$, and (d) are the allowed states when $\alpha(0)=1$. The transmittances consist of three screens containing a circle of $1 \mathrm{~mm}$ of radius. Each transmittance was placed on an oscillating mount. The amplitude oscillation is $0.5 \mathrm{~mm}$ and the frequency is of $300 \mathrm{~Hz}$. The results were obtained using the values $p=q=0.5$. The mean source generated with the allowed states is shown in (e).
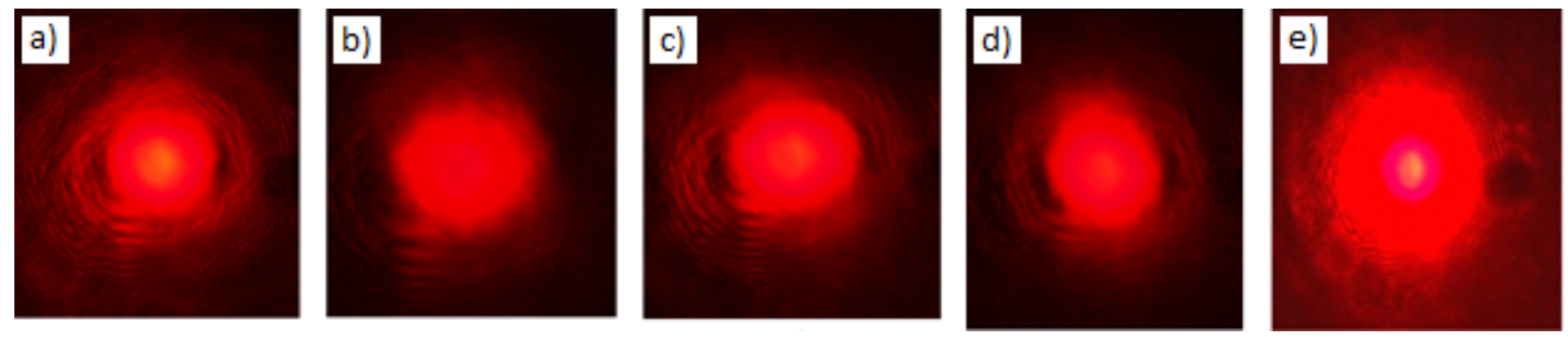

FIG. 6 Same motion states as it was described in Figure 5. (a),(b), (c), and (d) are the allowed states when $\alpha(0)=0$. These results were obtained when $p=q=0.5$. The mean source generated with the allowed states is shown in (e).

From Eq. (10) is easy to show that the transition probabilities must be

$$
P\left(X_{2}=0 \mid X_{1}=0\right)=P\left(X_{3}=0 \mid X_{2}=0\right)=(1-p) .
$$

Of course, this process has an associated intensity $\left\langle I_{c}\right\rangle$ which is the intensity value for the completely coherent field. The all set of possible values for the triple correlation are obtained using Eq. (10) and it is shown in Table 1. We remark that each correlation has a mean intensity value associated with it, which depends on the initial state $(\alpha(0)$ or $\alpha(1))$ and on the transition probabilities $(p, q)$.

In general all the mean intensity values for each state listed in Table 1 are different because depends on the movement state of each transmittance. With this set of irradiance values, we can calculate the mean intensity of the secondary source. If the experiment is performed $S$ times, it means that we expect that the intensity $I_{000}$ appears $\alpha(0)(1-p)^{2} S$ times, the intensity $I_{010}$ appears $\alpha(0) p q S$ times and so on for the other intensities.

For the experimental development, the transmittances are placed on individual oscillating mounts and each one consists in a screen containing a circle of approximately $0.5 \mathrm{~mm}$ of diameter. The kind of movements are harmonic oscil- lations perpendicular to the optical axis, with frequencies range [200-1000] $\mathrm{Hz}$ and amplitude oscillations in the range [0.1-0.5] $\mathrm{mm}$. The process starts by illuminating the first transmittance for a time $t_{0}$, after this time, the motion state of the second transmittance is obtained according to the transition probabilities $(p, q)$. If the first transmittance is static, the probability value $p$ of the motion state of the second transmittance is assigned; otherwise, we assign the probability value $q$. In a similar way we assign the probabilities of the motion state of the third transmittance.

The probability transitions are implemented with a sequential computer algorithm, following the tree graph sketched in Figure $4(\mathrm{~b})$. The motion parameters are driven with a signal generator and the mean intensity values were registered with a ccd detector, in order to have an intensity function dependent on position. To associate a physical meaning to Table 1, we identified two simple cases. The first case is obtained when $\alpha(0)=1$, which means that the first transmittance is static and only the states allowed are $\{(0,0,0),(0,1,0),(0,0,1),(0,1,1)\}$. The second case occurs when the first transmittance is mobile, that is $\alpha(0)=0$. The corresponding allowed states are given by $\{(1,0,0),(1,0,1),(1,1,0),(1,1,1)\}$. The corresponding experimental results are shown in Figure 5 and 6 . Also in these figures we 
show the mean intensity of the source, generated with these four states. To obtain the mean intensity we use the values $p=q=0.5$, this implies that all the states are equally probable.

By comparing the experimental results given by Figure 5(e) and Figure 6(e) is evident that the two sources have mean intensities completely different. These results make evident the dependence of the source structure with the probability $\alpha(0)$ to the motion state for the control transmittance. This means that selecting different values of these probability values, the temporal evolution of the source goes by different paths, generating different mean intensity values.

The experimental results shown in Figure 5 and 6 are for the values $\alpha(0)=1$ and $\alpha(0)=0$. The general case is obtained when is in the interval [0,1], and the eight possible states may be present, when the experiment is performed many times the probabilistic values for each state, listed in table becomes evident in the occurrence number, generating different mean intensities to the secondary source. This occurs when the process is stabilized, this happen when the experiment is performed $S$ times, being $S$ a large natural number. Then the probability effects are reflected on the occurrence of each state. From Table 1 , we have that the state $P(0,0,0)$ occurs $\alpha(0)(1-p)^{2} S$ times, the state $P(0,1,0)$ occurs $\alpha(0) p q S$ times and so on for the other states.

From Eq. (9), the correlation coefficient is rewritten as

$$
\begin{aligned}
\gamma(x, y, z, p, q, \alpha(0)) \\
\quad=\left(\frac{P(0,0,0) I_{c}(x, y, z)-\sum_{n=1}^{7} P_{n}(i, j, k) I_{i j k}(x, y, z)}{P(0,0,0) I_{c}(x, y, z)+\sum_{n=1}^{7} P_{n}(i, j, k) I_{i j k}(x, y, z)}\right),
\end{aligned}
$$

where the state $P(0,0,0)$ must be excluded in the sum, and $I_{i j k}(p)$ corresponds to the irradiance on a point $(x, y, z)$ associated to the state $P(i, j, k)$. Eq. (18) shows that the coherence correlation coefficient depends on the transition probabilities $(p, q)$ and the probability $\alpha(0)$ of the initial state of motion to the first transmittance.

\section{CONCLUSIONS}

We presented a method to synthesize Markovian sources whose intensity distribution and coherence parameters are easily tuneable. The study was made by using a separable transmittance that it is formed by the product of individual transmittances. The separability implies that the set of components is suitable to be motion correlated; this allows us to induce a convolution function with time-dependent phase function. The induced coherence features are manifested by changes in its radiometric features. The motions of the transmittances are time-ordering, and the light emission from the secondary source corresponds with a Markov chain. The motions between transmittances are synchronized by means of a conditional probability density function $\rho\left(t_{1}, t_{2}, \ldots, t_{k} \mid t_{k+1}, t_{k_{2}}, \ldots, t_{N}\right)$, and the time evolution depends on the motion state of the first transmittance which is consider as the control transmittance. Consequently the radiometric features and coherence parameters evolve by different paths. For the design of the sources, we are free to select the transition probability values $(p, q)$ and the initial probability of the motion state of the first transmittance. The kind of transmittances and parameters motion offers the possibility to generate a great variety of sources. The influence of these possible values is reflected in the mean intensity of the source.

\section{A PROPAGATION OF COHERENCE FEATURES}

An interesting point of the analysis is to describe the propagation of the coherence parameters. An accurate representation for the amplitude function on an arbitrary point $(x, z)$ is obtained from the angular spectrum model

$$
\phi(x, z)=\int_{-\infty}^{\infty} T(u) e^{i 2 \pi(x u+z p)} d u
$$

where $(u, p)$ are the spatial frequencies, $T(u)$ is the Fourier transform of the transmittance function. In a previous paper [15], we prove that the amplitude correlation function between two arbitrary points $(X 1, X 2)$, is given by

$$
W\left(X_{1}, X_{2}\right)=\left\langle\phi\left(X_{1}\right) \phi^{*}\left(X_{2}\right)\right\rangle=\int_{\infty}^{\infty}\left\langle|T(u)|^{2}\right\rangle e^{i 2 \pi X \cdot U} d U,
$$

where the square brackets express the expectation value of the power spectrum whose variables satisfies $X=\left(x_{2}-x_{1}, z_{2}-z_{1}\right)$ and $U=(u, p)$. The coherence function for transmittance in harmonic movements according to Eq. (4), take the form

$$
\begin{aligned}
W\left(X_{1}, X_{2}\right)= & \int\langle| T_{1}(u) e^{i 2 \pi u f_{1}(t)} \otimes T_{2}(u) e^{i 2 \pi u f_{2}(t)} \otimes \ldots \\
& \left.\left.\otimes T_{n}(u) e^{i 2 \pi u f_{n}(t)}\right|^{2}\right\rangle e^{i 2 \pi(X \cdot U)} d U
\end{aligned}
$$

where $X=(x, z)$ and $x=x_{2}-x_{1}, z=z_{2}-z_{1}$, it must be noted that in the particular case that $z_{2}=z_{1}$, Eq. (A.3) acquires the form of a Fourier transform according with the Van-Cittert Zernike theorem. For the case of the two transmittances in harmonic movement previously presented, we find that the coherence function takes the form

$$
\begin{aligned}
& W\left(X_{1}, X_{2}, t\right) \\
& \quad=\int_{-\infty}^{\infty}\langle I(u, z=0)\rangle e^{i 2 \pi\left(\left(x_{2}-x_{1}\right) u+\left(z_{2}-z_{1}\right) p\right)} d u,
\end{aligned}
$$

endequation substituting the expression for the mean intensity given by Eq. (8), we obtain

$$
\begin{aligned}
W\left(X_{1}, X_{2}\right)= & \iint_{-\infty}^{\infty}\left\langle T_{1}(h) T_{2}(h-u) T_{1}^{*}(s) T_{2}^{*}(s-u)\right\rangle \\
& \times M(h, s, u) e^{i 2 \pi\left(\left(x_{2}-x_{1}\right) u+\left(z_{2}-z_{1}\right) p\right)} d h d s d u,
\end{aligned}
$$

where $M(h, s, u)$ satisfies Eq. (7). The Eq. (A.5) represents the coherence distribution on arbitrary points having as a boundary condition the mean intensity of the source. 


\section{References}

[1] A. H. Dhalla, J. V. Migacz, and J. A. Izatt, "Crosstalk rejection in parallel optical coherence tomography using spatially incoherent illumination with partially coherent sources," Opt. Lett. 35, 2305-2307 (2010).

[2] J. A. Izatt, M. R. Hee, G. M. Owen, E. A. Swanson, and J. G. Fujimoto, "Optical coherence microscopy in scattering media," Opt. Lett. 19, 590-592 (1994).

[3] T. Xie, Z. Wang, and Y. Pan, "High-speed optical coherence tomography using fiberoptic acousto-optic phase modulation," Opt. Express 11, 3210-3219 (2003).

[4] T. Brixner, I. V. Stiopkin, and G. R. Fleming, "Tunable twodimensional femtosecond spectroscopy," Opt. Lett. 29, 884-886 (2004).

[5] G. Martínez-Niconoff, P. Martínez-Vara, J. Muñoz-Lopez, J. C. Juarez-Morales, and A. Carbajal-Dominguez, "Partially coherent surface plasmon modes," J. Europ. Opt. Soc. Rap. Public. 6, 11009 (2011).

[6] L. Mandel, and E. Wolf, Optical coherence and quantum optics (Cambridge University Press, Cambridge, 1995).

[7] A. S. Ostrovsky, G. Martínez-Niconoff, V. Arrizón, P. Martínez-Vara, M. A. Olvera-Santamaría, and C. Rickenstorff-Parrao, "Modulation of coherence and polarization using liquid cristal spatial light modulators," Opt. Express 17, 5257-5264 (2009).

[8] G. Martínez-Niconoff, J. C. Ramírez San Juan, J. Muñoz López, and P. Martínez Vara, "Incoherent convergence of diffraction free fields," Opt. Commun. 275, 10-13 (2007).
[9] T. Anna, S. K. Dubey, C. Shakher, A. Roy, and D. S. Mehta, "Sinusoidal fringe projection system based on compact and non-mechanical scanning low-coherence Michelson interferometer for three-dimensional shape measurement," Opt. Commun. 282, 1237-1242 (2009).

[10] N. Nakajima, "Phase retrieval using an aperture-array filter under partially coherent illumination," Opt. Commun. 282, 2128-2135 (2009).

[11] G. Martínez-Niconoff, J. Carranza-Gallardo, and A. CornejoRodriguez, "Synthesis of tunable moiré patterns," Opt. Commun. 126, 29-33 (1996).

[12] J. W. Goodman, Introduction to Fourier optics (McGraw-Hill companies, New York, 2000).

[13] G. F. Arfken, and H. J. Weber, Mathematical methods for physicists (Academic Press, San Diego, 2001).

[14] G. N. Watson, A treatise on the theory of Bessel functions (Cambridge University Press, Cambridge, 1966).

[15] G. Martínez-Niconoff, P. Martínez-Vara, and A. CarbajalDominguez, "Eikonal equation for partially coherent fields," Opt. Commun. 259, 488-491 (2006).

[16] J. W. Goodman, Statistical optics (Wiley-Interscience, New York, 1985).

[17] P. H. Hoel, S. C. Port, and Ch. J. Stone, Introduction to stochastic processes (Houghton Mifflin Company, Boston, 1972)

[18] L. Wasserman, All of statistics (Springer, New York, 2004). 